\title{
An Analysis of Derivational Affixes in Commencement speech By Steve Jobs
}

\author{
Dedi Rahman Nur \\ Widya Gama Mahakam University \\ d.blues84@gmail.com \\ Ainul Kirom \\ Widya Gama Mahakam University \\ ainulkirom@gmail.com
}

\begin{abstract}
The study expects to an investigation of derivational appends in the content of initiation discourse by Steve Jobs. The essayist utilized the majority of the words that were connected prefix and postfix as the information. The information sources were all content of initiation discourse by Steve Jobs. This study utilizing subjective plan and substance investigation approach. The aftereffect of the study demonstrated that there were 78 all out words in the content of Commencement discourse which joined derivational fastens. The study discovered 69 postfixes and 9 prefixes. The foundations of the words that has been grouped in light of the grammatical feature are 17 (descriptor), 27 (thing), 33 (verb), 1 (adverb).From the finish of this study, the author proposes that to enhance their dominance of vocabulary, the perusers ought to apply the derivational fastens by breaking the word into its components root and attaches in light of the fact that from single word they can get the structure of words and them additionally discover how the words fabricated. By knowing the roots, the perusers can assemble the word without anyone else's input
\end{abstract}

Keywords: affix, derivational, prefix, suffix.

\section{INTRODUCTION}

Language is often defined as a means of communication. It offers an idea that when everyone or everything is conducting communication, language, then, takes place as the medium of communication among the participants. With one's language can convey or receive information from others. As Finegan (2008) stated that the language is often viewed as a vehicle of thought, a system of expression that mediates the transfer of thought from one person to another. Considering the importance of English, the government has included it as one of the compulsory subjects to be taught to the student. In the newest of Indonesia education curriculum 2013, English is taught at school starting from the junior high school up to university. English lessons will be very influential for the development of the Indonesian state.

Furthermore, studying vocabulary becomes an important aspect in acquiring English and it is not easy to master it. As Heibert \& Kamil (2005) say that vocabulary is not a 
developmental skill or one that can ever be seen as fully mastered. The expansion and elaboration of vocabularies is something that extends across a lifetime. Vocabulary is the knowledge of meanings of words. What complicates this definition is the fact that words come in at least two forms: oral and print. Knowledge of words also comes in at least two forms, receptive - that which we can understand or recognize-and productive-the vocabulary we use when we write or speak. Another theory says that vocabulary is an essential part of language learning and the question of how much vocabulary a learner needs to know to achieve a particular purpose remains an important area of research and discussion. In other words, it is important to study about vocabulary because when we are learning a foreign language, and we have lack of the vocabulary, we will find the difficulties in understanding the meaning of the language itself.

On the contrary, if we have enough vocabulary it will make us easier to learn a new language and to understand the meaning of the language itself. There are some ways to enrich our vocabulary. One of them is by reading. It can be by the reading book, short story, newspaper, fable or speech. So in this analysis, the researchers use Commencement speech by Steve Jobs as the data source, because from this speech we can increase our knowledge, and there are many derivational affixes. Steve Jobs was an American businessman. He was best known as the co-founder, chairman, and chief executive officer (CEO) of Apple Inc.; CEO and largest shareholder of Pixar Animation Studios; a member of The Walt Disney Company's board of directors following its acquisition of Pixar; and founder, chairman, and CEO of NeXT Inc. Jobs is widely recognized as a pioneer of the microcomputer revolution of the 1970s, along with Apple co-founder Steve Wozniak. Shortly after his death, Jobs's official biographer, Walter Isaacson, described him as the "creative entrepreneur whose passion for perfection and ferocious drive revolutionized six industries: personal computers, animated movies, music, phones, tablet computing, and digital publishing.

Particularly, what the researchers investigated in this research was regarding with derivational affixes which is related to Morphology. In this case, Lieber (2009) said that Morphology is the study of word formation, including the ways new words are coined in the languages of the world, and the way forms of words are varied depending on how they're used in sentences. Somehow related to this research, the investigation of derivational affixes reflected in Commencement Speech by Steve Jobs is hopefully can enrich students' vocabulary and build better achievement on English skills proficiency.

Script Journal Volume 1, Issue 1, April 2016 || ISSN 2477-1880 || E-ISSN 2502-6623 http://jurnal.fkip-uwgm.ac.id/index.php/Script 


\section{METHODOLOGY}

The research method is qualitative with the design of the study is Derivational Affixes Analysis. Creswell (2009) states that qualitative inquirers use theory in their studies in several ways. This research also belongs to descriptive study since it just collected and analyzed the derivational affixes in the text of Commencement speech by Steve Jobs. The Subject of the study is photographs, art objects, videotapes, or any forms of sound (Creswell, 2009). Here the researchers use Steve Jobs speech entitled Commencement speech. The researchers, in this case use the review document to get information. The researchers analyzed the text of the Commencement speech by Steve Jobs.

The source of data for this study is a text of commencement speech by Steve Jobs. The data of the study was obtained from the internet. "Data" refers to a collection of organized information, usually the result of experience, observation, experiment This may consist of numbers, words, or images, particularly as measurements or observations of a set of variables (Yin, 2011). The data in this study was collected using following steps; the first researchers choose the speech. Second, the researchers listened and downloaded the text of the speech. Third, the researchers Found and Listing all the word contains derivational affixes.

In order to check the data validity, the researchers used Triangulation to validate regarding with the data found. Triangulation is aimed to check the result of interpretation by a researchers to relevant sources or theories, peer group interpretation, and comparison to other researchers' findings (Bogdan \& Biklen, 1992). In addition, there are four basic types of triangulation pointed out by Miles \& Huberman (1992) as follows:

a. Data triangulation: the use of variety of data sources in a study.

b. Investigator triangulation: the use of several different researchers or evaluators.

c. Theory triangulation: the use of multiple perspectives to interpret a single set of data.

d. Methodological triangulation: the use of multiple methods to study a single problem.

To conclude, the researchers used all of basic types of Triangulation above to check the data found since they are needed to check the data validity.

\section{FINDING}

The objective of this research was to know the derivational affixes in Commencement speech by Steve Jobs and the words itself consist of root or base added by derivational affix 
that has been changed the part of speech. The research data was taken from all the text of the Commencement speech by Steve Jobs (Peestandingup, 2006). After watching the video and reading the text of the Commencement speech by Steve Jobs, in this study researchers showed that there were 78 total words in the text of Commencement speech which attached derivational affixes. The study found 69 suffixes and 9 prefixes.

\section{DISCUSSION}

After presenting the words which are found in the text of Commencement speech by Steve Jobs (News.stanford.edu, 2005), the researchers presents the table which is going to show the derivational affixes. This table below is going to show the words, bases or roots, part of speech, derivational affixes, and note which is founding the text of Commencement speech by Steve Jobs. This research uses coding for makes it easy for readers. The coding P1.L1 it was meant paragraph 1 line 1, P1.L2 it was meant paragraph 1 line 2, P2.L1 it was meant paragraph 2 line 1, P2.L2 it was meant paragraph 2 line 2 and etc..

\begin{tabular}{|c|c|c|c|c|c|c|c|}
\hline \multirow{2}{*}{ No } & \multirow{2}{*}{$\begin{array}{c}\text { Word } \\
(\mathrm{P}=\text { Paragraph }) \\
(\mathrm{L}=\text { Line })\end{array}$} & \multirow{2}{*}{$\begin{array}{l}\text { Part of } \\
\text { Speech }\end{array}$} & \multirow{2}{*}{ Roots } & \multirow{2}{*}{$\begin{array}{l}\text { Part of } \\
\text { Speech }\end{array}$} & \multicolumn{2}{|c|}{$\begin{array}{c}\text { Derivational } \\
\text { Affixes }\end{array}$} & \multirow{2}{*}{ Note } \\
\hline & & & & & Prefix & Suffix & \\
\hline (1) & (2) & (3) & (4) & (5) & (6) & (7) & (8) \\
\hline 1 & $\begin{array}{c}\text { Commencement } \\
\text { (P1. L1) }\end{array}$ & Noun & Comment & Verb & & -cement & $\begin{array}{l}\text { Noun } \\
\text { Maker }\end{array}$ \\
\hline 2 & $\begin{array}{c}\text { Graduation } \\
\text { (P1.L3) }\end{array}$ & Noun & Graduate & Verb & & -ion & $\begin{array}{l}\text { Noun } \\
\text { Maker }\end{array}$ \\
\hline 3 & $\begin{array}{l}\text { Biological } \\
\text { (P4.L9) }\end{array}$ & Adj. & Biology & Noun & & -cal & $\begin{array}{l}\text { Adj. } \\
\text { Maker }\end{array}$ \\
\hline 4 & $\begin{array}{l}\text { Unwed } \\
\text { (P4.L9) }\end{array}$ & Adj. & Wed & Verb & Un- & & $\begin{array}{l}\text { Adj. } \\
\text { Maker }\end{array}$ \\
\hline 5 & $\begin{array}{c}\text { Adoption } \\
(\mathrm{P} 4 . L 10) \\
\end{array}$ & Noun & Adopt & Verb & & -ion & $\begin{array}{l}\text { Noun } \\
\text { Maker }\end{array}$ \\
\hline 6 & $\begin{array}{l}\text { Strongly } \\
(\mathrm{P} 4 . L 11) \\
\end{array}$ & Adv. & Strong & Adj. & & $-1 y$ & $\begin{array}{l}\text { Adv. } \\
\text { Maker }\end{array}$ \\
\hline 7 & $\begin{array}{l}\text { Really } \\
\text { (P4.L14) }\end{array}$ & Adv. & Real & Adj. & & $-1 y$ & $\begin{array}{l}\text { Adv. } \\
\text { Maker }\end{array}$ \\
\hline 8 & $\begin{array}{l}\text { Middle } \\
\text { (P4.L15) }\end{array}$ & Noun & Mid & Adj. & & -le & $\begin{array}{l}\text { Noun } \\
\text { Maker }\end{array}$ \\
\hline 9 & $\begin{array}{l}\text { Unexpected } \\
\text { (P4.L15) }\end{array}$ & Adj. & Expect & Verb & Un- & -ed & $\begin{array}{c}\text { Adj. } \\
\text { Maker }\end{array}$ \\
\hline 10 & $\begin{array}{l}\text { Refused } \\
\text { (P4.L18) }\end{array}$ & Adj. & Refuse & Verb & & $-d$ & $\begin{array}{l}\text { Adj. } \\
\text { Maker }\end{array}$ \\
\hline 11 & Expensive & Adj. & Expense & Noun & & -ive & Adj. \\
\hline
\end{tabular}

Script Journal Volume 1, Issue 1, April 2016 || ISSN 2477-1880 || E-ISSN 2502-6623

http://jurnal.fkip-uwgm.ac.id/index.php/Script 


\begin{tabular}{|c|c|c|c|c|c|c|c|}
\hline \multirow{2}{*}{ No } & \multirow{2}{*}{$\begin{array}{c}\text { Word } \\
(\mathrm{P}=\text { Paragraph }) \\
(\mathrm{L}=\text { Line })\end{array}$} & \multirow{2}{*}{$\begin{array}{l}\text { Part of } \\
\text { Speech }\end{array}$} & \multirow{2}{*}{ Roots } & \multirow{2}{*}{$\begin{array}{l}\text { Part of } \\
\text { Speech }\end{array}$} & \multicolumn{2}{|c|}{$\begin{array}{c}\text { Derivational } \\
\text { Affixes }\end{array}$} & \multirow{2}{*}{ Note } \\
\hline & & & & & Prefix & Suffix & \\
\hline & (P5.L23) & & & & & & Maker \\
\hline 12 & $\begin{array}{c}\text { Scary } \\
\text { (P5.L29) }\end{array}$ & Adj. & Scare & Noun & & $-y$ & $\begin{array}{l}\text { Adj. } \\
\text { Maker }\end{array}$ \\
\hline 13 & $\begin{array}{l}\text { Decision } \\
\text { (P5.L29) }\end{array}$ & Noun & Decide & Verb & & -ion & $\begin{array}{l}\text { Noun } \\
\text { Maker }\end{array}$ \\
\hline 14 & $\begin{array}{l}\text { Romantic } \\
\text { (P6.L32) }\end{array}$ & Adj. & Romance & Noun & & -ic & $\begin{array}{l}\text { Adj. } \\
\text { Maker }\end{array}$ \\
\hline 15 & $\begin{array}{l}\text { Across } \\
\text { (P6.L34) }\end{array}$ & Adv. & Cross & Verb & & & $\begin{array}{l}\text { Adv. } \\
\text { Maker }\end{array}$ \\
\hline 16 & $\begin{array}{c}\text { Every } \\
\text { (P6.L34) }\end{array}$ & Adj. & Ever & Adv. & & & $\begin{array}{l}\text { Adj. } \\
\text { Maker }\end{array}$ \\
\hline 17 & $\begin{array}{l}\text { Curiosity } \\
\text { (P6.L36) }\end{array}$ & Noun & Curious & Adj. & & - ty & $\begin{array}{l}\text { Noun } \\
\text { Maker }\end{array}$ \\
\hline 18 & $\begin{array}{l}\text { Intuition } \\
\text { (P6.L36) }\end{array}$ & Noun & Intuit & Verb & & -ion & $\begin{array}{l}\text { Noun } \\
\text { Maker }\end{array}$ \\
\hline 19 & $\begin{array}{l}\text { Priceless } \\
\text { (P6.L37) }\end{array}$ & Adj. & Price & Noun & & -less & $\begin{array}{l}\text { Adj. } \\
\text { Maker }\end{array}$ \\
\hline 20 & $\begin{array}{l}\text { Instruction } \\
\text { (P7.L38) }\end{array}$ & Noun & Instruct & Verb & & -ion & $\begin{array}{l}\text { Noun } \\
\text { Maker }\end{array}$ \\
\hline 21 & $\begin{array}{c}\text { Drawer } \\
(\text { P7.L40) }\end{array}$ & Noun & Draw & Verb & & -er & $\begin{array}{l}\text { Noun } \\
\text { Maker }\end{array}$ \\
\hline 22 & $\begin{array}{l}\text { Beautifully } \\
\text { (P7.L40) }\end{array}$ & Adv. & Beauty & Noun & & -fully & $\begin{array}{l}\text { Adv. } \\
\text { Maker }\end{array}$ \\
\hline 23 & $\begin{array}{l}\text { Amount } \\
\text { (P7.L43) }\end{array}$ & Noun & Mount & Verb & $a-$ & & $\begin{array}{l}\text { Noun } \\
\text { Maker }\end{array}$ \\
\hline 24 & $\begin{array}{c}\text { Combinations } \\
\text { (P7.L43) }\end{array}$ & Noun & Combine & Verb & & -tion & $\begin{array}{l}\text { Noun } \\
\text { Maker }\end{array}$ \\
\hline 25 & $\begin{array}{l}\text { Beautiful } \\
\text { (P7.L44) }\end{array}$ & Adj. & Beauty & Noun & & - ful & $\begin{array}{l}\text { Adj. } \\
\text { Maker }\end{array}$ \\
\hline 26 & $\begin{array}{c}\text { Historical } \\
(\mathrm{P} 7 . L 44)\end{array}$ & Adj. & History & Noun & & -cal & $\begin{array}{l}\text { Adj. } \\
\text { Maker }\end{array}$ \\
\hline 27 & $\begin{array}{l}\text { Practical } \\
\text { (P8.L46) }\end{array}$ & Adj. & Practice & Noun & & -al & $\begin{array}{l}\text { Adj. } \\
\text { Maker }\end{array}$ \\
\hline 28 & $\begin{array}{l}\text { Application } \\
\text { (P8.L46) }\end{array}$ & Noun & Apply & Verb & & -tion & $\begin{array}{l}\text { Noun } \\
\text { Maker }\end{array}$ \\
\hline 29 & $\begin{array}{l}\text { Proportionally } \\
\text { (P8.L51) }\end{array}$ & Adv. & Proportion & Noun & & -ally & $\begin{array}{l}\text { Adv. } \\
\text { Maker }\end{array}$ \\
\hline 30 & $\begin{array}{c}\text { Likely } \\
\text { (P8.L52) }\end{array}$ & Adj. & Like & Verb & & $-1 y$ & $\begin{array}{c}\text { Adj. } \\
\text { Maker }\end{array}$ \\
\hline 31 & $\begin{array}{l}\text { Personal } \\
\text { (P8.L52) }\end{array}$ & Adj. & Person & Noun & & -al & $\begin{array}{l}\text { Adj. } \\
\text { Maker }\end{array}$ \\
\hline 32 & Wonderful & Adj. & Wonder & Verb & & -ful & Adj. \\
\hline
\end{tabular}




\begin{tabular}{|c|c|c|c|c|c|c|c|}
\hline \multirow{2}{*}{ No } & \multirow{2}{*}{$\begin{array}{c}\text { Word } \\
(\mathrm{P}=\text { Paragraph }) \\
(\mathrm{L}=\text { Line })\end{array}$} & \multirow{2}{*}{$\begin{array}{l}\text { Part of } \\
\text { Speech }\end{array}$} & \multirow{2}{*}{ Roots } & \multirow{2}{*}{$\begin{array}{l}\text { Part of } \\
\text { Speech }\end{array}$} & \multicolumn{2}{|c|}{$\begin{array}{c}\text { Derivational } \\
\text { Affixes }\end{array}$} & \multirow{2}{*}{ Note } \\
\hline & & & & & Prefix & Suffix & \\
\hline & (P8.L54) & & & & & & Maker \\
\hline 33 & $\begin{array}{l}\text { Impossible } \\
\text { (P8.L55) }\end{array}$ & Adj. & Impose & Verb & & -ble & $\begin{array}{l}\text { Adj. } \\
\text { Maker }\end{array}$ \\
\hline 34 & $\begin{array}{l}\text { Difference } \\
\text { (P9.L61) }\end{array}$ & Noun & Different & Adj. & & & $\begin{array}{l}\text { Noun } \\
\text { Maker }\end{array}$ \\
\hline 35 & $\begin{array}{c}\text { Lucky } \\
\text { (P11.L63) }\end{array}$ & Adj. & Luck & Noun & & $-y$ & $\begin{array}{l}\text { Adj. } \\
\text { Maker }\end{array}$ \\
\hline 36 & $\begin{array}{c}\text { Early } \\
\text { (P11.L63) }\end{array}$ & Adv. & Ear & Noun & & $-1 y$ & $\begin{array}{l}\text { Adv. } \\
\text { Maker }\end{array}$ \\
\hline 37 & $\begin{array}{c}\text { Finest } \\
\text { (P11.L66) }\end{array}$ & Adj. & Fine & Noun & & $-s t$ & $\begin{array}{l}\text { Adj. } \\
\text { Maker }\end{array}$ \\
\hline 38 & $\begin{array}{l}\text { Creation } \\
\text { (P11.L67) }\end{array}$ & Noun & Create & Verb & & -ion & $\begin{array}{l}\text { Noun } \\
\text { Maker }\end{array}$ \\
\hline 39 & $\begin{array}{c}\text { Earlier } \\
\text { (P11.L67) }\end{array}$ & Adj. & Ear & Noun & & -lier & $\begin{array}{l}\text { Adj. } \\
\text { Maker }\end{array}$ \\
\hline 40 & $\begin{array}{l}\text { Diverge } \\
\text { (P11.L71) }\end{array}$ & Verb & Verge & Noun & Di- & & $\begin{array}{l}\text { Verb } \\
\text { Maker }\end{array}$ \\
\hline 41 & $\begin{array}{l}\text { Eventually } \\
\text { (P11.L71) }\end{array}$ & Adv. & Event & Noun & & -ally & $\begin{array}{l}\text { Adv. } \\
\text { Maker }\end{array}$ \\
\hline 42 & $\begin{array}{l}\text { Directors } \\
\text { (P11.L72) }\end{array}$ & Noun & Direct & Adj. & & -or & $\begin{array}{c}\text { Noun } \\
\text { Maker }\end{array}$ \\
\hline 43 & $\begin{array}{l}\text { Publicly } \\
\text { (P11.L73) }\end{array}$ & Adv. & Public & Noun & & $-1 y$ & $\begin{array}{l}\text { Adv. } \\
\text { Maker }\end{array}$ \\
\hline 44 & $\begin{array}{l}\text { Generation } \\
(\mathrm{P} 12 . \mathrm{L} 76)\end{array}$ & Noun & Generate & Verb & & -ion & $\begin{array}{l}\text { Noun } \\
\text { Make }\end{array}$ \\
\hline 45 & $\begin{array}{l}\text { Apologize } \\
\text { (P12.L78) }\end{array}$ & Verb & Apology & Noun & & -ize & $\begin{array}{l}\text { Verb } \\
\text { Maker }\end{array}$ \\
\hline 46 & $\begin{array}{c}\text { Badly } \\
\text { (P12.L78) }\end{array}$ & Adv. & $\mathrm{Bad}$ & Adj. & & $-1 y$ & $\begin{array}{l}\text { Adv. } \\
\text { Maker }\end{array}$ \\
\hline 47 & $\begin{array}{c}\text { Failure } \\
\text { (P12.L78) }\end{array}$ & Noun & Fail & Verb & & - re & $\begin{array}{l}\text { Noun } \\
\text { Maker }\end{array}$ \\
\hline 48 & $\begin{array}{c}\text { Slowly } \\
(\mathrm{P} 12 . L 80)\end{array}$ & Adv. & Slow & Adj. & & $-1 y$ & $\begin{array}{l}\text { Adv. } \\
\text { Maker }\end{array}$ \\
\hline 49 & $\begin{array}{l}\text { Heaviness } \\
\text { (P13.L84) }\end{array}$ & Noun & Heavy & Adj. & & -ness & $\begin{array}{l}\text { Noun } \\
\text { Maker }\end{array}$ \\
\hline 50 & $\begin{array}{l}\text { Successful } \\
\text { (P13.L85) }\end{array}$ & Adj. & Success & Noun & & -ful & $\begin{array}{l}\text { Adj. } \\
\text { Maker }\end{array}$ \\
\hline 51 & $\begin{array}{l}\text { Replaced } \\
(\text { P13.L85) }\end{array}$ & Verb & Place & Noun & $\mathrm{Re}-$ & $-d$ & $\begin{array}{l}\text { Verb } \\
\text { Maker }\end{array}$ \\
\hline 52 & $\begin{array}{l}\text { Lightness } \\
\text { (P13.L85) }\end{array}$ & Noun & Light & Adj. & & -ness & $\begin{array}{l}\text { Noun } \\
\text { Maker }\end{array}$ \\
\hline
\end{tabular}




\begin{tabular}{|c|c|c|c|c|c|c|c|}
\hline \multirow{2}{*}{ No } & \multirow{2}{*}{$\begin{array}{c}\text { Word } \\
(\mathrm{P}=\text { Paragraph }) \\
(\mathrm{L}=\text { Line })\end{array}$} & \multirow{2}{*}{$\begin{array}{l}\text { Part of } \\
\text { Speech }\end{array}$} & \multirow{2}{*}{ Roots } & \multirow{2}{*}{$\begin{array}{l}\text { Part of } \\
\text { Speech }\end{array}$} & \multicolumn{2}{|c|}{$\begin{array}{c}\text { Derivational } \\
\text { Affixes }\end{array}$} & \multirow{2}{*}{ Note } \\
\hline & & & & & Prefix & Suffix & \\
\hline 53 & $\begin{array}{l}\text { Beginner } \\
(\mathrm{P} 13 . L 85)\end{array}$ & Noun & Begin & Verb & & -er & $\begin{array}{l}\text { Noun } \\
\text { Maker }\end{array}$ \\
\hline 54 & $\begin{array}{l}\text { Creative } \\
\text { (P13.L86) }\end{array}$ & Adj. & Create & Verb & & -ive & $\begin{array}{l}\text { Adj. } \\
\text { Maker }\end{array}$ \\
\hline 55 & $\begin{array}{l}\text { Animation } \\
\text { (P14.L91) }\end{array}$ & Noun & Animate & Verb & & -ion & $\begin{array}{l}\text { Noun } \\
\text { Maker }\end{array}$ \\
\hline 56 & $\begin{array}{l}\text { Remarkable } \\
\text { (P14.L92) }\end{array}$ & Adj. & Mark & Verb & & -able & $\begin{array}{l}\text { Adj. } \\
\text { Maker }\end{array}$ \\
\hline 57 & $\begin{array}{c}\text { Truly } \\
\text { (P15.L102) }\end{array}$ & Adv. & True & Adj. & & $-1 y$ & $\begin{array}{l}\text { Adv. } \\
\text { Maker }\end{array}$ \\
\hline 58 & $\begin{array}{c}\text { Settle } \\
\text { (P15.L104) }\end{array}$ & Verb & Set & Noun & & $-l e$ & $\begin{array}{l}\text { Verb. } \\
\text { Maker }\end{array}$ \\
\hline 59 & $\begin{array}{l}\text { Relationship } \\
\text { (P15.L105) }\end{array}$ & Noun & Relate & Verb & & -ionship & $\begin{array}{l}\text { Noun } \\
\text { Maker }\end{array}$ \\
\hline 60 & $\begin{array}{l}\text { Impression } \\
\text { (P17.L111) } \\
\end{array}$ & Noun & Impress & Verb & & -ion & $\begin{array}{l}\text { Noun } \\
\text { Maker }\end{array}$ \\
\hline 61 & $\begin{array}{l}\text { Expectation } \\
\text { (P18.L118) }\end{array}$ & Noun & Expect & Verb & & -tion & $\begin{array}{l}\text { Noun } \\
\text { Maker }\end{array}$ \\
\hline 62 & $\begin{array}{l}\text { Embarrassment } \\
\text { (P18.L119) }\end{array}$ & Noun & Embarrass & Verb & & -ment & $\begin{array}{l}\text { Noun } \\
\text { Maker }\end{array}$ \\
\hline 63 & $\begin{array}{c}\text { Avoid } \\
(\mathrm{P} 18 . \mathrm{L} 121) \\
\end{array}$ & Verb & Void & Noun & a- & & $\begin{array}{l}\text { Verb } \\
\text { Maker }\end{array}$ \\
\hline 64 & $\begin{array}{c}\text { Already } \\
\text { (P18.L122) }\end{array}$ & Adv. & Ready & Adj. & Al- & & $\begin{array}{l}\text { Adv. } \\
\text { Maker }\end{array}$ \\
\hline 65 & $\begin{array}{c}\text { Clearly } \\
\text { (P19.L125) }\end{array}$ & Adv. & Clear & Adj. & & $-1 y$ & $\begin{array}{l}\text { Adv. } \\
\text { Maker }\end{array}$ \\
\hline 66 & $\begin{array}{l}\text { Incurable } \\
\text { (P19.L127) }\end{array}$ & Adj. & Incur & Verb & & -able & $\begin{array}{l}\text { Adj. } \\
\text { Maker }\end{array}$ \\
\hline 67 & $\begin{array}{c}\text { Curable } \\
(\mathrm{P} 20 . \mathrm{L} 140)\end{array}$ & Adj. & Cure & Verb & & -able & $\begin{array}{l}\text { Adj. } \\
\text { Maker }\end{array}$ \\
\hline 68 & $\begin{array}{l}\text { Certainty } \\
\text { (P21.L143) }\end{array}$ & Adv. & Certain & Adj. & & $-1 y$ & $\begin{array}{l}\text { Adv. } \\
\text { Maker }\end{array}$ \\
\hline 69 & $\begin{array}{c}\text { Useful } \\
\text { (P21.L143) }\end{array}$ & Adj. & Use & Verb & & - ful & $\begin{array}{l}\text { Adj. } \\
\text { Maker }\end{array}$ \\
\hline 70 & $\begin{array}{l}\text { Intellectual } \\
\text { (P21.L144) }\end{array}$ & Adj. & Intellect & Noun & & -ual & $\begin{array}{l}\text { Adj. } \\
\text { Maker }\end{array}$ \\
\hline 71 & $\begin{array}{l}\text { Invention } \\
\text { (P22.L148) }\end{array}$ & Noun & Invent & Verb. & & -ion & $\begin{array}{l}\text { Noun } \\
\text { Maker }\end{array}$ \\
\hline 72 & $\begin{array}{l}\text { Gradually } \\
\text { (P22.L150) }\end{array}$ & Adv. & Gradual & Adj. & & $-1 y$ & $\begin{array}{l}\text { Adv. } \\
\text { Maker }\end{array}$ \\
\hline 73 & $\begin{array}{c}\text { Dramatic } \\
(\mathrm{P} 22 . L 151) \\
\end{array}$ & Adj. & Drama & Noun & & $-\mathrm{tic}$ & $\begin{array}{l}\text { Adj. } \\
\text { Maker }\end{array}$ \\
\hline
\end{tabular}




\begin{tabular}{|c|c|c|c|c|c|c|c|}
\hline \multirow{2}{*}{ No } & Word & Part of & Roots & $\begin{array}{c}\text { Part of } \\
\text { Speech }\end{array}$ & Prefix & Suffix & Affixes \\
\cline { 6 - 8 } & $\begin{array}{c}\text { P = Paragraph) } \\
\text { (L= Line) }\end{array}$ & Speech & & Note & \\
\hline 74 & $\begin{array}{c}\text { Opinions } \\
\text { (P23.L154) }\end{array}$ & Noun & Opine & Verb & & -ion & $\begin{array}{c}\text { Noun } \\
\text { Maker }\end{array}$ \\
\hline 75 & $\begin{array}{c}\text { Publication } \\
\text { (P24.L158) }\end{array}$ & Noun & Public & Adj. & & -tion & $\begin{array}{c}\text { Noun } \\
\text { Maker }\end{array}$ \\
\hline 76 & $\begin{array}{c}\text { Idealistic } \\
\text { (P24.L165) }\end{array}$ & Adj. & Ideal & Noun & & -tic & $\begin{array}{c}\text { Adj. } \\
\text { Maker }\end{array}$ \\
\hline 77 & $\begin{array}{c}\text { Adventurous } \\
\text { (P25.L170) }\end{array}$ & Adj. & Adventure & Noun & & -ous & $\begin{array}{c}\text { Adj. } \\
\text { Maker }\end{array}$ \\
\hline 78 & $\begin{array}{c}\text { Anew } \\
\text { (P25.L173) }\end{array}$ & Adv. & New & Adj. & a- & & $\begin{array}{c}\text { Adv. } \\
\text { Maker }\end{array}$ \\
\hline
\end{tabular}

Table 1 Derivational Affixes (Commencement speech by Steve Jobs)

As the objective of the research which is to found the derivational affixes and the bases or roots of the words in text of Commencement speech by Steve Jobs, the table above is showing how to find out the derivational affixes and the roots. It can be seen that the words in text of Commencement speech by Steve Jobs are analyzed by separating the derivational affixes and the roots, so it was clearly obtained derivational affixes and the roots of the words.

From the table above, analyzing the structure of words, there were derivational affixes that can be found in text of Commencement speech by Steve Jobs It will be shown in the table below

\begin{tabular}{|c|c|c|c|}
\hline \multirow{2}{*}{ No } & \multicolumn{2}{|c|}{ Derivational Affixes } & \multirow{2}{*}{$\begin{array}{c}\text { The Number of Derivational } \\
\text { Affixes }\end{array}$} \\
\cline { 2 - 3 } & Prefix & Suffix & 1 \\
\hline 1 & Al- & & 1 \\
\hline 2 & Un- & & 2 \\
\hline 3 & Re- & & 4 \\
\hline 4 & A- & & 11 \\
\hline 5 & Di- & -ion & 1 \\
\hline 6 & & -ic & 4 \\
\hline 7 & & -tion & 1 \\
\hline 8 & & -ous & 1 \\
\hline 9 & & -ty & 11 \\
\hline 10 & & - -ly & 1 \\
\hline 11 & & - -ize & 4 \\
\hline 12 & & - -ul & 3 \\
\hline 13 & & - -able & 2 \\
\hline 14 & & -ness & 3 \\
\hline 15 & & $-y$ & \\
\hline 16 & & & \\
\hline
\end{tabular}

Script Journal Volume 1, Issue 1, April 2016 || ISSN 2477-1880 || E-ISSN 2502-6623

http://jurnal.fkip-uwgm.ac.id/index.php/Script 


\begin{tabular}{|c|c|c|c|}
\hline \multirow{2}{*}{ No } & \multicolumn{2}{|c|}{ Derivational Affixes } & \multirow{2}{*}{$\begin{array}{c}\text { The Number of Derivational } \\
\text { Affixes }\end{array}$} \\
\hline & Prefix & Suffix & \\
\hline 17 & & -al & 2 \\
\hline 18 & & $-c e$ & 1 \\
\hline 19 & & -ive & 2 \\
\hline 20 & & -ual & 1 \\
\hline 21 & & -cement & 1 \\
\hline 22 & & -cal & 2 \\
\hline 23 & & $-\mathrm{le}$ & 2 \\
\hline 24 & & -ed & 1 \\
\hline 25 & & $-d$ & 2 \\
\hline 26 & & -less & 1 \\
\hline 27 & & -er & 2 \\
\hline 28 & & -fully & $\sqrt{3}$ \\
\hline 29 & & -ally & 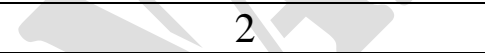 \\
\hline 30 & & -ble & 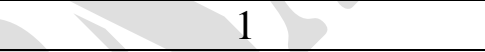 \\
\hline 31 & & -st & 1 \\
\hline 32 & & -lier & 1 \\
\hline 33 & & -or & 1 \\
\hline 34 & & -re & 1 \\
\hline 35 & & -ionship & 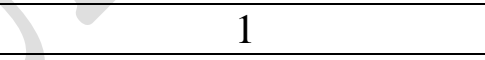 \\
\hline 36 & & -ment & 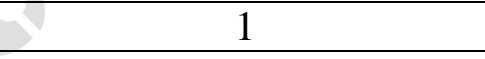 \\
\hline 37 & & -tic & 2 \\
\hline
\end{tabular}

Table 2 The Number Derivational Affixes

From the table 4.2, it show that there al- (1), un- (1), re- (2), a- (4), di- (1), -ion (11), ic (1), -tion (4), -ous (1), -ty (1), -ly (11), -ize (1), -ful (4), -able (3), -ness (2), -y (3), -al (2), ce (1), -ive (2), -ual (1), -cement (1), -cal (2), -le (2), -ed (1), -d (2), -less (1), -er (2), -fully (1), -ally (2), -ble (1), -st (1), -lier (1), -or (1), -re (1), -ionship (1), -ment (1), -tic (2) and little derivational affixes found in Commencement speech by Steve Jobs. Most frequently present only suffix -ion and -lyas many as 11 times.

From the table above, the researchers also obtained the root of the words in Commencement speech by Steve Jobs. They are 17 (adjective), 27 (noun), 33 (verb), 1 (adverb). It shows that the part of speech classification of the bases or roots in Commencement speech by Steve Jobs is mostly presented in verb.

After analyzing the derivational affixes, the researchers found that derivational affixes also have the function such as verb maker, noun maker, adjective maker and adverb maker found in Commencement speech by Steve Jobs. It was shown in the table below. 


\begin{tabular}{|l|l|l|}
\hline No. & The Function of Derivational Affixes & $\begin{array}{l}\text { The Number of The Function of } \\
\text { Derivational Affixes }\end{array}$ \\
\hline 1 & Verb Maker & 5 \\
\hline 2 & Noun Maker & 28 \\
\hline 3 & Adjective Maker & 29 \\
\hline 4 & Adverb Maker & 16 \\
\hline
\end{tabular}

Table 3 The Number of The Function of Derivational Affixes

From the table above, it showed that there are verb maker (5); noun maker (28); adjective maker (29); and adverb maker (16) as the function of derivational affixes found in Commencement speech by Steve Jobs. It showed that Adjective maker which is most frequently present

\section{CONCLUSION}

Based on the findings of the study elaborated in chapter IV, the researchers concluded several conclusions.

In this study researchers showed that there were 78 total words in text of Commencement speech which attached derivational affixes. The study found 69 suffixes and 9 prefixes. Derivational affixes in text of Commencement speech by Steve Jobs are al- (1), un- (1), re- (2), a- (4), di- (1), -ion (11), -ic (1), -tion (4), -ous (1), -ty (1), -ly (11), -ize (1), ful (4), -able (3), -ness (2), -y (3), -al (2), -ce (1), -ive (2), -ual (1), -cement (1), -cal (2), -le (2), -ed (1), -d (2), -less (1), -er (2), -fully (1), -ally (2), -ble (1), -st (1), -lier (1), -or (1), -re (1), -ionship (1),-ment (1), -tic (2).The roots from the words that has been classified based on the part of speech are 17 (adjective), 27 (noun), 33 (verb), 1 (adverb). From the conclusion of this study, the researchers suggests that to improve their mastery of vocabulary, the readers should be apply the derivational affixes by breaking the word into its elements root and affixes because from one word they can get the structure of words and they also find how the words built. By knowing the roots, the readers can build the word by themselves.

The function of derivational affixes in text of Commencement speech by Steve Jobs was noun maker (28), verb maker (5), adjective maker (29), and adverb maker (16). Commencement speech by Steve Jobs, the bases or roots of words that had been classified into the part of speech were16 (adjective), 28 (noun), 33 (verb) and 1 (adverb). 


\section{BIBLIOGRAPHY}

Bogdan, R. C., \& Biklen, S. K. (1992). Qualitative Research for Education: An Introduction to Theory and Methods. Boston, MA: Allyn and Bacon.

Creswell, J. W. (2009). Research Design Qualitative, Quantitative, and Mixed Methods Approaches. California: Sage Publication, Inc.

Finegan, E. (2008). Language: Its Structure and Use. New York: Thomson Higher Education.

Heibert, E., \& Kamil, M. (2005). Teaching and Learning Vocabulary. New Jersey: Lawrence Erlbaum Associates, Inc.

Lieber, R. (2009). Introducing Morphology. New York: Cambridge University Press.

Miles, M. ., \& Huberman, A. M. (1992). Analysis Data Kualitatif”. Translated by Tjetjep Rohendi Rohidi from "Qualitative Data Analysis ". Jakarta: UI-Press.

News.stanford.edu. (2005). Text Commencement speech. Retrieved September 20, 2015, from http://news.stanford.edu/news/2005/june15/jobs-061505/

Peestandingup. (2006). Steve Jobs Stanford Commencement Speech 2005. Retrieved September 9, 2016, from http://www.youtube.com/watch?v=D1R-jKKp3NA

Yin, R. K. (2011). Qualitative Research from Start to Finish. New York: A Division of Guildford Publications, Inc. 